\title{
What makes the Mueller a liar: A multiple-cue approach
}

\author{
PAMELA G. McCLELLAN, IRA H. BERNSTEIN and CALVIN P. GARBIN \\ University of Texas at Arlington, Arlington, Texas
}

\begin{abstract}
Subjects made magnitude estimations of fins-in and fins-out Mueller Lyer stimuli. Shaft length, fin length, and fin angle were within-subjects variables, and the sitmuli could be either conventional solid drawings or dot forms, a between-subjects variable. The parametric effects of these variables were similar to results previously obtained. However, principal emphasis was given to lens model and related multivariate analyses of the subjects' judgments. These analyses suggested that no single "critical" cue seemed to account for the illusion. Rather, various sets of cues could be formulated to simulate the subjects' judgmental behavior equivalently. In addition, field-independent subjects showed better knowledge of the cue structure than field-dependent subjects. In the main, subjects in this experiment performed like subjects have in tasks not involving visual illusions with multiple sources of information varying in their relevance.
\end{abstract}

What is commonly known as the multiple-cue paradigm is derived from Brunswik's $(1952,1956)$ interest in how subjects achieve knowledge of a distal stimulus when the criterial dimension is quite poorly related to individual cues in the proximal stimulus. This paradigm gives rise to a pair of important, but contrasting, findings: (1) Judgments can achieve the criterial dimension quite well despite the relatively small amount of valid information in the individual cues, and (2) cues are often used quite inappropriately compared to optimal information combination, thus preventing judgments from being as accurate as they might be. The term "inappropriate" can subsume several distinct forms of judgmental error, which include interstimulus or intertrial inconsistency, or the failure to adhere to one set of rules for all occasions, and use of the wrong (nonoptimal) cues or combinations of cues. Goldberg (1970) discussed these errors of judgment in the context of clinical judgments. He illustrated how clinical psychologists often make trait judgments, for example, maladjustment, using MMPI protocols or the like in a complex, configurational manner. However, such judgments can be shown to correlate more poorly with the trait being judged than can simpler indices.

Multiple-cue research has stimulated considerable interest in the application of multivariate models to perception and cognition. The model most often, but not exclusively, employed to this end is Brunswik's (1956) lens model (also see Hammond, Hursch, \& Todd, 1964;

This paper is based in part upon a dissertation submitted by the senior author to the second author in partial fulfillment of the doctoral requirement at the University of Texas at Arlington. The authors are indebted to an anonymous referee for comments useful in structuring the underlying issues. Requests for reprints should be sent to Ira H. Bernstein, Psychology Department, University of Texas at Arlington, P.O. Box 19528, Arlington, TX 76019.
Hammond \& Summers, 1972; Tucker, 1964; Szucko \& Kleinmuntz, 1981). One role these multivariate models serve is to provide a regression equation to infer the subject's decision structure (e.g., Einhorn, Kleinmuntz, \& Kleinmuntz, 1979).

The multiple-cue paradigm might initially appear different from what we will term the "illusion paradigm." In the illusion paradigm, the criterial dimension is well represented in the proximal stimulus, but there is other, misleading information that the subject uses in addition to the criterial dimension, thus producing judgmental error. Perhaps the most popular, but not sole, example of this paradigm is the visual geometric illusion. By definition, all illusions imply a judgmental inaccuracy that would not occur in the absence of this other information, for example, the fins and their associated geometrical parameters, which define Mueller-Lyer stimuli and produce the illusion. Consequently, major interest is normally focused upon what corresponds to the second of the findings obtained in multiple-cue studies, the use of inappropriate cues. In this case, however, misuse means the inclusion of illusion-producing information. Multivariate models allow one to determine how that information is incorporated into the subject's judgment.

These two paradigms may initially be perceived as different because there is a difference in the relationship of the criterial dimension to the other information, as seen from the perspective of the experimenter. In the multiplecue situation, each individual cue can potentially make a unique, and usually positive (helpful), contribution to veridical perception. In the illusion situation, one feature of the proximal stimulus is usually sufficient to represent the criterial dimension of the distal stimulus. The remaining information may usually be regarded as misleading and as contributing to nonveridical perception. Nonetheless, an overriding similarity between the multiple-cue and 
illusion paradigms is that subjects misuse information in both cases. Although the word "cue"' has not typically been used to describe the noncriterial information in an illusion, we will use that term because it is descriptive from the subject's standpoint and so describes what we feel is a fundamental similarity between the two paradigms. Our consequent interest is in what cues are inappropriately combined with the criterial dimension in an illusion and how they are combined as a step towards discovering why.

Multivariate models have been used to infer cue utilization in the illusion situation, although, to our knowledge, these have not specifically involved the lens model, and have not employed geometric illusions as stimuli. Hake, Faust, McIntyre, and Murray (1967) obtained size judgments of test squares. In the control condition, the test square appeared by itself on each trial. In one of the experimental conditions, the test square appeared within a slightly larger square whose size did not change over trial. Performance was more accurate in this condition than in the control condition, reflecting the often noted superiority of comparative judgments over absolute judgments. By contrast, two additional experimental conditions employed an outer square that varied randomly over trials, the two conditions differing in the amount of variation in the outer square. Performance was generally poorer in these latter two conditions than in the control condition.

The particular multivariate technique used (linear discriminant analysis) revealed that different factors were responsible for judgmental error in different classes of subjects. Some subjects simply used the distance between the test and outer square as their basis for judgment. Others judged the two squares separately and used the size of the outer square as a control or covariate in judging the inner square. Hake, Rodwan, and Weintraub (1966) suggested a reason why subjects might use information that misleads them. Responding to such information can produce a subjective dimension of judgment in which there is more apparent variation than the veridical dimension. In the authors' words, subjects met a criterion of coherence, or internal stability of judgments, in lieu of accuracy, or conformance to the experimenter's demands. We see this as a useful way to look at geometrical illusions.

More recently, Bernstein (e.g., Bernstein, Fisicaro, \& Fox, 1976; Bernstein, Smith, \& Adey, 1977) used multivariate modeling to study visual masking and metacontrast. They found that the weightings given cues employed by the subject changes systematically as the stimulus onset asynchrony separating test and mask increased but this change was quite different from that expected from the lateral inhibitory models then prevailing.

Although visual masking is not usually conceived of as geometric illusion, it does share the characteristic impairment produced by the addition of irrelevant and misleading contextual visual information. Moreover, similar mechanisms have been suggested as relevant to the study of masking and visual illusions. Coren and Girgus (1978) have discussed the role that lateral inhibition has served to explain geometric illusions, and forms of lateral inhibition have provided popular, though debatable, explanations of visual masking (Breitmeyer \& Ganz, 1976). This paper will not be concerned with the general role of lateral inhibition or other physiological mechanisms in perception, however.

The heuristic value of multivariate models to perception led us to consider their applicability to the most popular geometric illusion, the Mueller-Lyer. Particular emphasis was placed upon the following issue. Several authors have proposed that the illusion can be explained by a single critical stimulus, defined as some function of the basic geometry of the figure, for example, its shaft length, fin length, or fin angle. The hypothesized role of intertip distance (ITD) in Pressey's (1967; Pressey \& Bross, 1973; Pressey \& Murray, 1976) model is one example of a critical stimulus approach. Another example is Virsu's (1971, also see Richards \& Kaufman, 1969, and Kaufman \& Richards, 1969) center of gravity approach.

It is possible that there is no critical stimulus, as Coren and Girgus's (1978) review would suggest on empirical grounds. Quite possibly, a Mueller-Lyer or other illusory figure could contain several distinguishable aspects, each of which is separately capable of producing misperception. For example, suppose one subject paid attention to ITD, but another paid attention to his or her efferent feedback from incipient eye movements. The former would behave in accord with assimilation theory; the latter would behave in accord with center of gravity theory. Both would exhibit the basic illusion, but for different reasons, paralleling what Hake et al.'s (1967) subjects did. Regression analysis provides one way to explore differences in the cues used across subjects. The search for the critical stimulus may thus be identifed with the search for a regression equation that is invariant across subjects and conditions. This may not be obtainable, as in the example above, because of intra- and interindividual differences in cue utilization.

Both the results of previous research with the MuellerLyer illusion and the logic of multiple regression suggest that it may not be possible to obtain a unique equation, even for a single subject in one condition. Although an experimenter can create a set of orthogonal independent variables, such as the basic ones presented above, they will be correlated with derived variables like ITD. Suppose the correlations with these derived variables are high, and the latter variables are included in the regression model. This is a situation known as "multicollinearity." When it is present, different regression equations, composed of different cues (or different weightings of the same cues) which imply different processing modes can fit the data equally well. Thus, it is usually difficult or impossible to choose among predictor models when there is multicollinearity.

Even though the multicollinearity issue is stated as a statistical problem, it is not necessarily artifactual. As Ein- 
horn et al. (1979) note, "the indeterminancy in estimating weights when cues are correlated parallels the organism's difficulty in the matter (p. 467)." Conversely, one can limit analysis to the experimentally defined predictors, since these will not be multicollinear. However, failure to incorporate a cue that the subject may have used will make it difficult to infer the subject's decisional strategy if the cue is not linearly derivable from those incorporated in the model. The ITD is an example of such a cue. This is a specific instance of what is known as the "specification" problem in multiple regression (Pedhazur, 1982). One deals with this problem by looking at alternative solutions.

In this study, we did emply the lens model among other multivariate tools. We used the experimentally defined cues of shaft length, fin length and fin angle, plus others to be noted in the Results section. Our evaluation of the critical-stimulus hypothesis will rest upon the assumptions made in regression analysis. These assumptions are similar to those made by Bernstein et al. $(1976,1977)$ and Hake et al. (1967) in the sense that differences between the use of discriminant function analysis and regression analysis are not relevant to the present discussion. Moreover, these assumptions are much less restrictive than those made in applying the lens model. The lens model was useful because it furnished a vocabulary and statistical procedures to use in a discussion of what we feel are basic issues. The lens model itself may be stated in the following form (Tucker, 1964):

$$
r_{a}=G_{e} R_{s}+C\left(1-R_{e}^{2}\right)^{.5}\left(1-R_{s}^{2}\right)^{.5}
$$

The lens model is a regression-based method of partitioning the correlation between the environment and judgments into various psychologically relevant components as described below. The subject's achievement $\left(r_{a}\right)$ is the observed correlation between the objective criterial dimension, or environment, which in the present case is shaft length, and the corresponding judgments of shaft length. It defines overall accuracy. In the present case, achievement and veridicality, that is, lack of illusion, are analogous concepts, except for the fact that the former is expressed in correlational units rather than as a mean deviation from shaft length.

The environmental linearity $\left(R_{e}\right)$ is the multiple correlation between the environment (physical shaft length) and the cues incorporated in the model. These cues may be limited to the three basic ones defined above, or they may also include derived cues such as the ITD. Alternative sets of cues define alternative lens models, which may be explored, as in any regression analysis, to provide the most parsimonious description. The $R_{\mathrm{e}}$ itself is a measure of the extent to which the criterial dimension may be represented in terms of a particular set of cues using multiple linear regression. Conversely, the judgmental linearity (or cognitive control, $\mathbf{R}_{\mathbf{s}}$ ) is the multiple correlation of judgments and cues. This is a measure of the extent to which the subject's bases for judgments may be represented in terms of the set of cues, again using multiple linear regression.

The correlations between individual cues, such as the ITD and the environment (physical shaft length), provide one way to define cue validities, which measure the importance of each cue when "predicting"' the environment. Similarly, the correlations between individual cues and judgments provide one way to define cue utilizations, which measure the importance of each cue when "predicting" the response. Thus, like the two measures they help define, $R_{e}$ and $R_{s}$, the cue validities and cue utilizations serve parallel functions on the stimulus and response side, respectively. Neither the cue validities nor the cue utilizations are explicitly represented in the lens model equation, but they are important byproducts of the underlying regression analysis.

The cue validities and cue utilizations may each be defined using indices other than correlations, for example, the predictor's beta weights in the respective multiple regression equations, among other indices. When the various cues vary independently of one another, as when the set of cues is limited to those that have been factorially manipulated, the various definitions of cue validity will be equivalent to one another (specifically, beta weights and correlations will be identical), as will the various definitions of cue utilization. This equivalence of importance measures will not be the case in most multiple regression analyses, since predictors need not be independent (Pedhazur, 1982). Wilkinson (1975) has illustrated how different measures of importance can lead to quite different conclusions when there is multicollinearity. The cue sets to be examined in this study are not necessarily independent; for example, we will incorporate ITD as a cue. Hence, we will be examining alternative definitions of cue "importance" on both the stimulus (cue validity) and response (cue utilization) side in our various analyses.

The subject's knowledge of the relationship (G) between cues and environment is the correlation between the linear components of the environment and the linear components of the judgments. It represents the similarity of the optimal weighting of the cue set. By contrast, $R_{s}$, or judgmental linearity, measures the degree of application of this knowledge to the judgments. It measures the extent to which judgments are determined by the linear weighting relative to other determinants, for example, randomness, nonlinear combinations of cues, and cues not incorporated into the model.

The configurational component $(\mathrm{C})$ is the correlation between the nonlinearities or residuals of the environment and judgments. The two quantities, $\mathrm{G}$ and $\mathrm{C}$, serve complementary roles. $G$ measures the extent to which judgments are based upon a linear combination of elements from the cue set, and $\mathrm{C}$ measures the extent to which judgments are based upon other information or nonlinear combinations derived from the cue set. Achievement is simply a weighted average of $C$ and $G$.

As noted above, sets of cues included in a particular lens model analysis can be defined in various ways. At 
the one extreme, we could limit the definition to the dimensions that were ostensibly manipulated in this experiment. At the other extreme, we could include such cues as ITD which are derived from this basic set, as long as they are not linearly dependent (perfectly predictable from other cues in the model). Suppose a subject were relying upon a derived variable such as ITD, and further suppose it correlated, at least to some extent, with physical shaft length (which it might or might not, depending upon how the variables were manipulated). Choice of elements included in the regression model (cue set) will not affect achievement $\left(r_{a}\right)$. However, it will affect the relative magnitudes of $\mathrm{G}$ and $\mathrm{C}$. A model that includes ITD will probably produce a higher value of $\mathrm{G}$ and a lower value of $C$ than a model that ignores ITD, if for no other reason than the bias inherent in multiple regression as predictors are added (Pedhazur, 1982). The cues correlated with ITD will account for judgments in part, but not in full, if these cues are not perfectly correlated with ITD.

Alternative models can thus be evaluated to determine which cues the subject employed. If $C$ is .0, the cues incorporated in the model will account for all criterionrelated variance in the subject's judgment (moreover, if $R_{s}$ is 1.0 , these cues will account for all judgmental variance, criterion-related or not). Thus, when $C$ is .0 , the set of cues is sufficient to describe the cues giving rise to the illusion (even if $R_{s}$ is less than 1.0). Inspection of the cue utilization measures can define which ones are necessary, except insofar as the cue set is multicollinear. Nonetheless, as will be shown, the lens model, and regression models in general, can be used to answer questions dealing with the invariance of judgmental criteria, specifically, the critical stimulus hypothesis. In no sense do we suggest that regression models are the only way to answer a very old question. What we do suggest is that they can provide a fresh approach to help decide whether the general issue of a critical stimulus is properly phrased or not.

If one defines physical shaft length as both the environment and one of the cues, $R_{e}$ becomes 1.0 and $C$ becomes indeterminate. This is not usually a problem in multiplecue studies because the cues can typically be made imperfect predictors of the environment $\left(\mathrm{R}_{\mathrm{e}}<1.0\right)$. Our solution to this problem was to define shaft length as a cue in subjective terms. That is, we used the subjects' length judgments of no-fin stimuli as the values of the shaft-length cue in the regression equation (physical shaft length defines the environment, of course). Since the correlation between judged shaft length and physical shaft length is not 1.0 , the degeneracy is avoided.

Issues apart from the critical-stimulus hypothesis were also investigated. Use of the lens model provides parameters that can help decide the extent to which the illusion arises from lack of knowledge of the linearities that are present (imperfect $G$ ) vs. failure to use this knowledge (imperfect $R_{s}$ ). In addition, we looked at the role of individual differences in performance on the Group
Embedded Figures Test (GEFT; Witkin, Oltman, Raskin, \& Karp, 1971), a measure of field independence (FI) vs. field dependence. Finally, we compared judgments of conventional solid drawings to dot forms, recognizing Coren, Girgus, Erlichman, and Hakstian's (1976) obtained differences.

\section{METHOD}

\section{Subjects}

Eleven males and 19 females were recruited from the introductory psychology subject pool and participated to fulfill a course requirement. Each subject was tested individually in sessions of approximately $50 \mathrm{~min}$ on each of 4 consecutive days. All subjects reported having normal or corrected-to-normal vision.

\section{Apparatus and Stimuli}

All stimuli were drawn in black ink on $102 \times 152 \mathrm{~mm}$ white cards. Contours were approximately $.5 \mathrm{~mm}$ in line figures, and dots were $.5 \mathrm{~mm}$ in diameter and $1.0 \mathrm{~mm}$ apart in dot figures. Shafts of all figures were centered both horizontally and vertically.

The stimuli weere presented on a three-field Gerbrands Harvard tachistoscope, Model T381. One field contained a central fixation point, the second contained the test figure, and the third contained a reference line. The viewing distance was $810 \mathrm{~mm}$.

Two sets of 128 stimulus cards were constructed, one of solid drawings and the other of dot forms. Each set represented the combination of four variables: (1) fin direction (fins in vs. fins out), (2) shaft length $\left(37,40,43\right.$, or $46 \mathrm{~mm}$, corresponding to visual angles of $2.6^{\circ}, 2.8^{\circ}$, $3.0^{\circ}$, and $3.2^{\circ}$, respectively), (3) fin angle $\left(20^{\circ}, 60^{\circ}, 100^{\circ}\right.$, or $140^{\circ}$, as measured between fins), and (4) fin length $(3,8,13$, or $18 \mathrm{~mm})$. In addition, four no-fin control stimuli $(37,40,43$, or $46 \mathrm{~mm})$ and 32 no-fin practice stimuli $(24.0$ to $64.5 \mathrm{~mm}$ ) were also constructed.

\section{Procedure}

We used the method of magnitude estimation because it would provide a larger number of discrete observations to compute necessary correlations than would more conventional methods. Each trial contained the following sequence: (1) the fixation point for $2 \mathrm{sec}$, (2) a 40-mm nofin comparison line for $1.3 \mathrm{sec}$, and, finally (3) the test stimulus for $1.3 \mathrm{sec}$. A $250-\mathrm{msec}$ interstimulus interval separated the three events. These durations were selected to make the judgment task comfortable, on the basis of the reports of two pilot subjects.

The subjects were told that the comparison line was 100 units long and were instructed to respond to each test by giving a judgment relative to that norm. The experimenter recorded the subject's response on that trial, inserted the next stimulus card, gave a verbal "ready" signal, and initiated the next trial.

The first session was devoted to practice, during which the subject received 160 trials, the 32 nó-fin control stimuli presented five times each in random order. This session served to familiarize the subjects with the magnitude estimation procedure and to determine whether they could discriminate among the stimuli. Four subjects who could not perform the task were eliminated at this point and replaced.

Each of the next two sessions involved 10 warm-up trials with the no-fin practice stimuli, followed by the 128 Mueller-Lyer and 4 no-fin control stimuli in random order. Presentation of the latter was constrained such that 2 of the no-fin lines appeared in the first 66 trials and 2 appeared in the last 66 trials. A rest period of 3 min followed every 33 trials. Each stimulus was judged twice in separate sessions.

The subjects were instructed to focus upon the fixation point once they heard the "ready signal." Both the fixation requirement and the short exposure durations were intended to minimize illusion decrements, as suggested by Eysenck and Slater (1958) and Lewis (1908). Although very short presentation times could have been used to eliminate eye movements, Erlebacher and Sekuler (1974) had found that fins-in, fins-out, and no-fin stimuli were differentially affected by very short exposure duration. The subjects were further instructed to try to keep their responses independent by basing them upon the " 100 unit" standard 
line and not upon previously seen test stimuli. The subjects rated each stimulus combination (4 fin lengths $\times 4$ fin angles $\times 4$ shaft lengths $\times 2$ fin directions) twice, once each in successive sessions.

The last session was devoted to administration of the GEFT. This was presented according to the directions in the test manual.

\section{RESULTS}

\section{Basic Parametric Analyses}

A preliminary analysis indicated that between-sessions differences and, consequently, illusion decrements were minimal. Data were therefore pooled over sessions. The standard deviations within cells (averaged over sessions) ranged from 2.8 to 17.3. In general, these standard deviations were correlated with shaft length and, to a lesser extent, other mean differences to be discussed. A fiveway analysis of variance (ANOVA) was then performed, with fin direction, shaft length, fin angle, and fin length as within-subjects variables and solid vs. dot forms (stimulus type) as a between-subjects variable.

The basic Mueller-Lyer effect, larger ratings to finsout stimuli than to fins-in stimuli, was obtained $[F(1,27)$ $=110.43, \mathrm{MSe}=911.58, \mathrm{p}<.001]$. The average ratings of fins-out, control (as obtained from a separate analysis), and fins-in stimuli were 112.1, 104.4 and 101.9. This pattern suggests the expansion produced by the finsout stimuli, relative to the control $(112.1-104.4$, or 7.7 units), was greater than contraction produced by the fins-in stimuli $(101.9-104.4$, or 2.5 , units). Judgements of shaft length increased with physical shaft length $[F(3,84)$

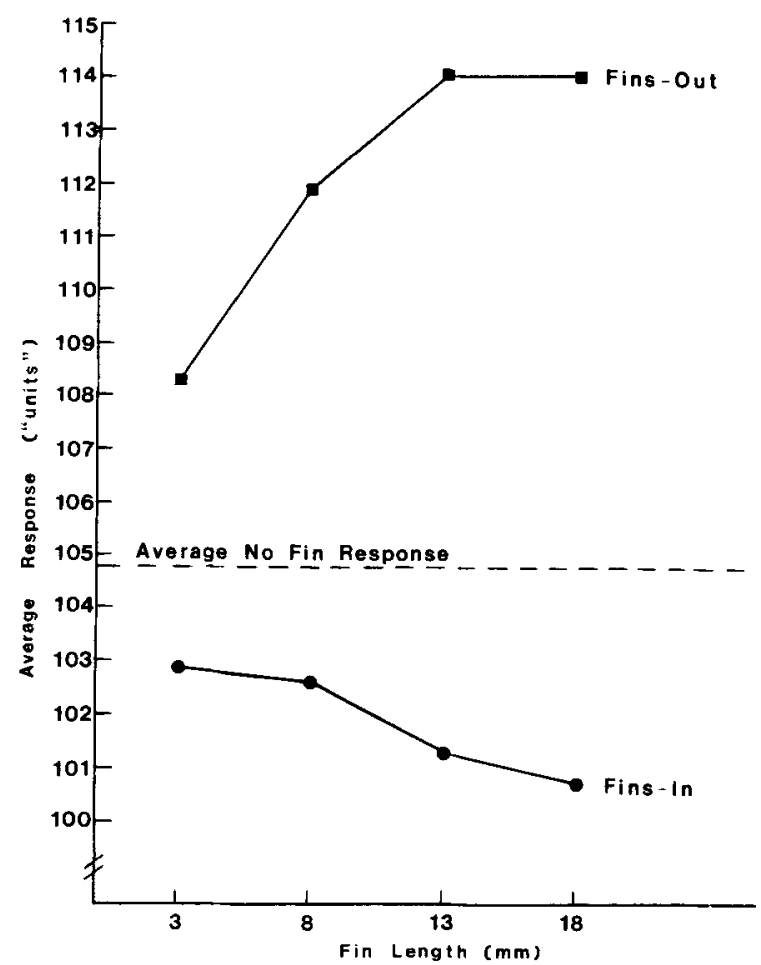

Figure 1. Average magnitude estimates as a function of fin length and fin direction.

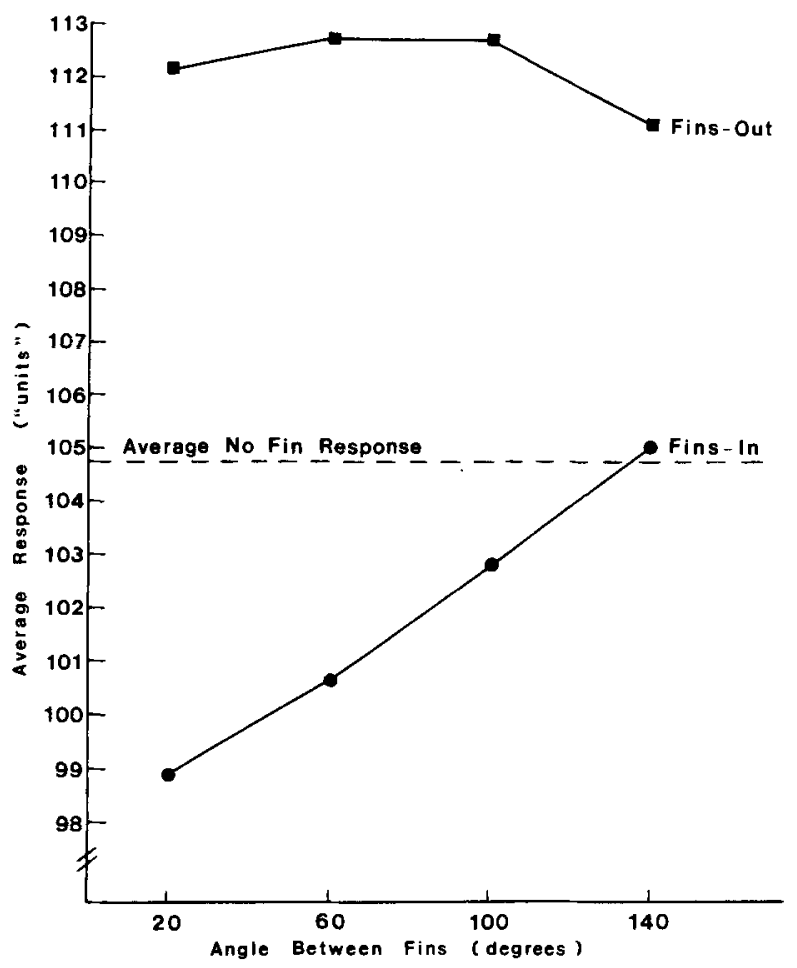

Figure 2. Average magnitude estimates as a function of fin angle and fin direction.

$=138.74, \mathrm{MSe}=283.46, \mathrm{p}<.001]$. The obtained averages as a function of increasing shaft length were $99.4,104.6,109.5$, and 111.4. This means that the magnitude estimation task was sensitive to the veridical aspects of perception.

Figure 1 illustrates the significant fin length $\times$ fin direction interaction $[\mathrm{F}(3,84)=49.08$, MSe $=10.83$., $\mathrm{p}<.001]$. Increasing fin length with fins-in stimuli induces a minimal, relatively linear decrease in judgments of shaft length. Increasing fin length with fins-out stimuli produces a relatively large change in judgments of shaft length up to $13 \mathrm{~mm}$, but makes relatively little difference beyond this point for the shaft lengths studied. The main effect of fin length is also significant, but it is of little importance because it is confounded with fin directions $[\mathrm{F}(3,84)=7.09, \mathrm{MSe}=25.59, \mathrm{p}<.001]$.

Figure 2 illustrates the significant fin angle $\times$ fin direction interaction $[\mathrm{F}(3,84)=36.41, \mathrm{MSe}=10.83$, $\mathrm{p}<.001$ ]. Judgments of shaft length increase with fin angle for fins-in stimuli, whereas there is essentially no effect of fin angle for fins-out stimuli. The main effect of fin angle is significant $[\mathrm{F}(3,84)=15.34$, MSe $=$ $266.10, p<.01]$, but this effect is also confounded with fin direction, and hence is of little importance.

Figure 3 illustrates the fin length $x$ fin angle $\times$ fin direction interaction, which was significant $[F(9,252)=$ 2.92, $\mathrm{MSe}=43.34, \mathrm{p}<.01]$, as was the fin length $\times$ fin angle interaction $[\mathrm{F}(9,252)=4.34, \mathrm{MSe}=89.85$, $\mathrm{p}<.001]$. Increasing fin angles caused increasing judg- 

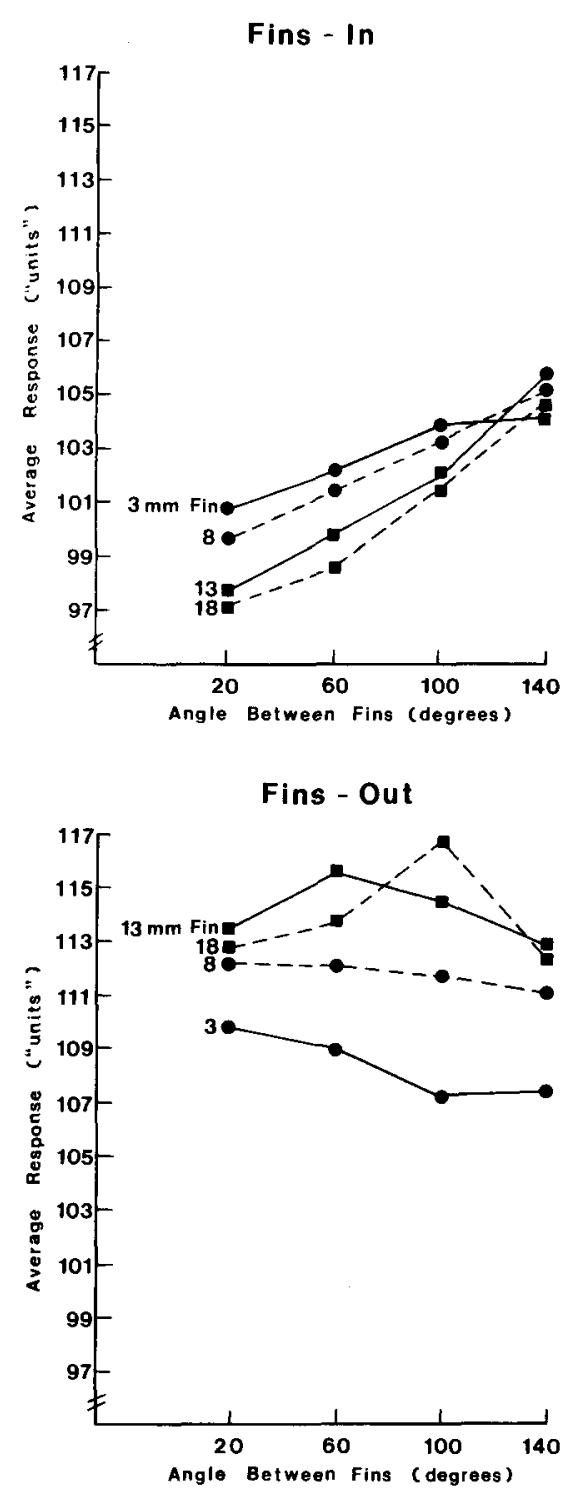

Figure 3. Average magnitude estimates as a function of fin length, fin angle, and fin direction.

ments of shaft length for all fin lengths with fins-in stimuli. These judgments terided to converge to a common point at the largest fin angle. Increasing fin angle had more complex effects with fins-out stimuli. It had relativley little effect with the two smallest fin lengths, whereas the relations were nonmonotonic with the two largest fin lengths. The fin length $\times$ fin angle $\times$ shaft length interaction also reached significance $[\mathrm{F}(27,756)=1.77, \mathrm{MSe}=25.91]$. Angle changes had little or no effect with short shaft lengths or short fin lengths. The fin angle effect increased as these two variables increased.

There was no main effect of stimulus type, but there were two interactions: stimulus type $\times$ fin angle, fin angle, $[\mathrm{F}(9,252)=4.34, \mathrm{MSe}=89.85, \mathrm{p}<.01]$ and stimulus type $\times$ fin angle $\times$ fin length $[F(9,252)=2.29$, $\mathrm{MSe}=39.31, \mathrm{p}<.05]$. Fin angle effects were greater for solid drawings than for the dot forms. In addition, the fin angle $X$ fin length interaction was quite pronounced with solid drawings, but nearly absent with dot forms. These interactions are shown in Figures 4 and 5, respectively.

The raw judgments were then transformed into relative deviations about the no-fin standard of the same length. This is one of several possible ways to see if the shaft length variable disappears when analysis is expressed as a deviation score. Although the shaft length effect was still present in this analysis, it was greatly reduced in magnitude $[F(3,84)=3.58, \mathrm{MSe}=.0004, \mathrm{p}<.05]$. This transformation does not alter other effects in the ANOVA. Similar results held when the data were expressed as deviations about the physical as opposed to the judged shaft length (no-fin control).

Finally, differences in response to the no-fin controls were essentially linear with respect to physical length and were equivalent for solid and dot fiures. The averages, with standard deviations in parentheses, pooled across subjects, sessions, and the two types of figures are 96.1 (3.4), $101.4(3,7), 107.3(4.4)$, and $113.0(6.5)$ for the 37-, 40-, 43-, and 46-mm lengths. The fact that this is linear, that is, fits a power function with slope of 1.0 , is consistent with Stevens (1962).

\section{Lens Model Analysis}

A lens model was constructed for each subject using the following cues, which were defined for each of the 128 experimental stimuli: (1) the judged length of the nofin control, (2) fin angle, (3) fin length, (4) ITD, and

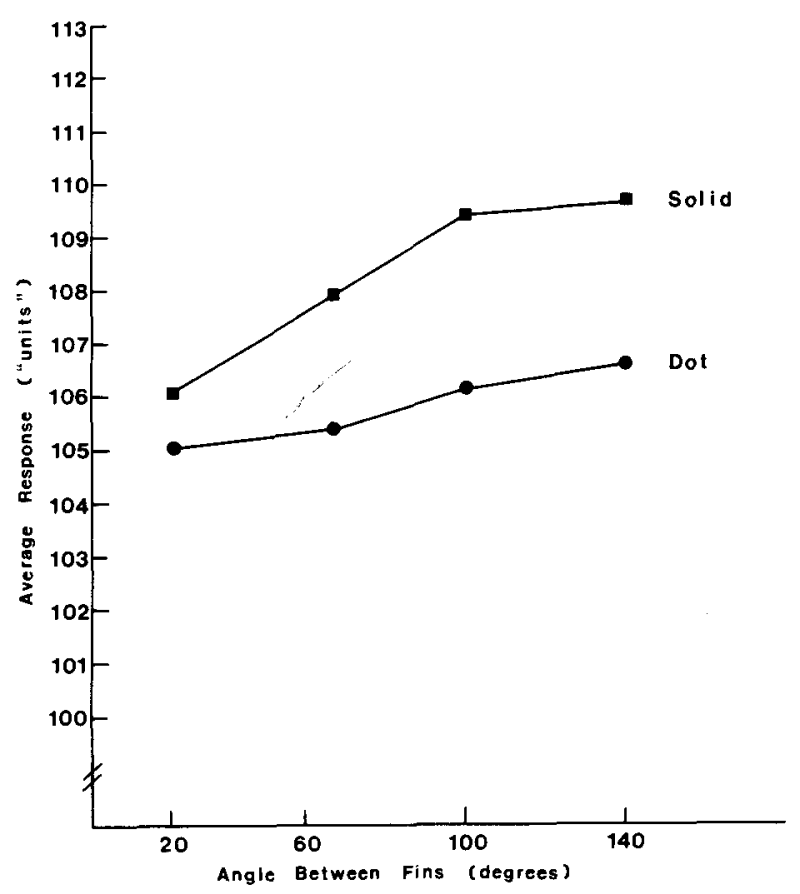

Figure 4. Average magnitude estimates as a function of fin angle and stimulus type (solid drawings vs. dot forms). 

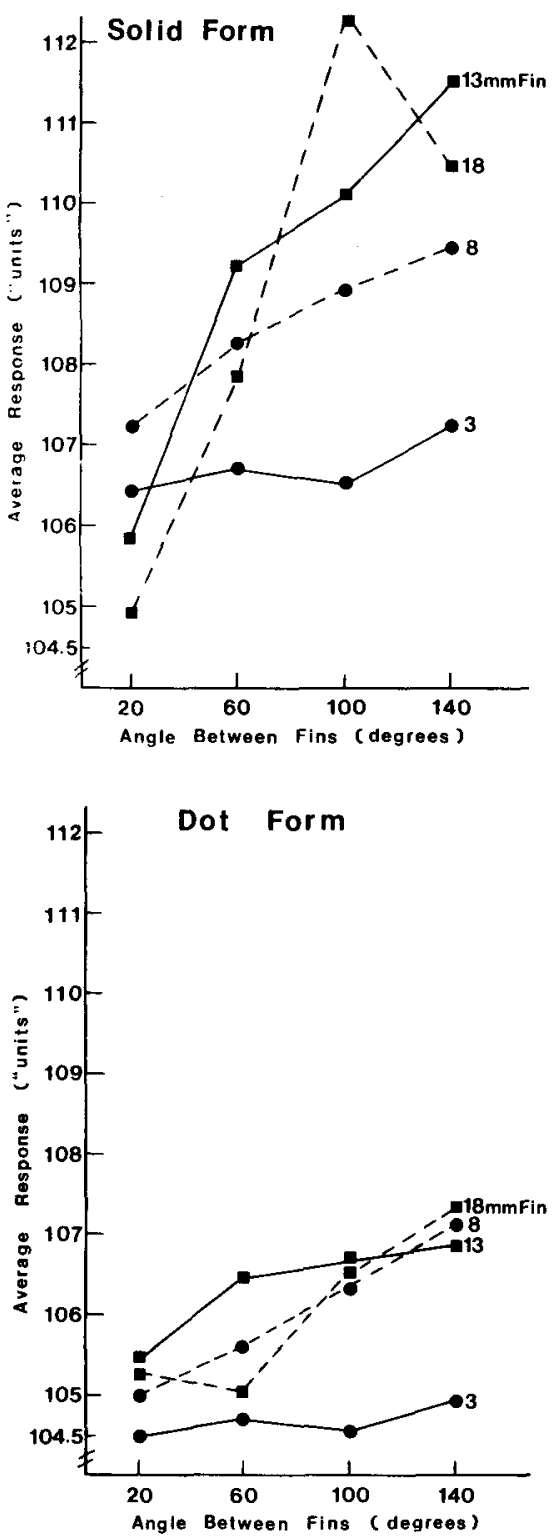

Figure 5. Average magnitude estimates as a function of fin length, fin angle, and stimulus type (solid drawings vs. dot forms).

(5) the vertical distance between the tip of the fin and the shaft $(\mathrm{H})$. The latter two measures may be derived from the remaining measures, but they are not linearly dependent and do not induce singularity in the underlying regression analyses. Singularity is a condition that can arise in multiple regression to preclude a solution (Pedhazur, 1982, pp. 780-781). Separate models were constructed for fins-in and fins-out stimuli and for the between-subjects factor, solid drawings vs. dot forms. The results obtained with this cue structure were equivalent to the results obtained with other cue structures.

Had there been no illusory effect, that is, had the fins not contributed to the judgments, only shaft length (whether physical or inferred from no-fin trials) would have had a nonzero beta weight. This is true even for such derived variables as ITD, which are correlated with shaftlength measures because beta weights describe the extent to which a change in a given predictor is associated with a change in the criterion (judged shaft length, in this case), holding constant other predictors. If a suitable shaft-length measure is sufficient as a cue, then, by definition, no other cue would vary with the criterion once shaft length was statistically controlled. Under these conditions, the beta weight for shaft length will be 1.0 in the absence of random judmental error or less than 1.0 in its presence.

Conversely, had the illusion been total in the sense that subjects responded totally to the fins and not to the shaft, the beta width for shaft-length measures would be .0 . The obtained results were intermediate to these extremes, as one would expect. That is, regardless of the model chosen, shaft length and fin parameters both had beta weights that differed significantly from .0 implying that veridical and illusory cues were representable in the judgment. Although this may not seem surprising, it need not be the case; a subject could conceivably respond solely to ITD or some other cue, in which case the beta weight for shaftlength measures would be .0 .

Because the no-fin cue differed for each subject, because it was based upon that subject's judgments, the environmental linearities $\left(R_{e}\right.$ or multiple correlations between the environment and cues) also differed among subjects. They were uniformly high, however, ranging from .88 to .997 , with a mean of .96 and a standard deviation of .03. These large magnitudes largely reflect the high correlation between physical shaft length and the nofin control measures.

The achievement $\left(r_{a}\right)$ ranged from .43 to .92 with a mean of .72 and a standard deviation of .10 . Knowledge of the linear relationship (G) between physical shaft length and its associated cues was slightly higher than the application of this knowledge $\left(\mathrm{R}_{\mathrm{s}}\right)$. The respective means were $.88(\mathrm{~s}=.08)$ and $.80(\mathrm{~s}=.08)$. The estimate of $R_{s}$ is probably inflated because there is slight multicollinearity (Pedhazur, 1982). None of these parameters varied as a function of fin direction or type of form.

The quantity $C$ is the correlation between variance in the environment and variance in the judgment that is not linearly derivable from the set of cues. As noted above, this is an index of the extent to which criterion-related cues were employed in a subject's judgment but not specified in the model. The obtained values were generally small, that is $<.40$ in 50 of 60 cases. This further suggests that the exceptional 10 subjects were employing a different judgmental strategy, which, by itself, is contrary to the critical-stimulus hypothesis.

\section{Cue Utilization Analysis}

We next looked at cue utilization by deriving various multiple regression equations linking judgments to differently defined cue sets. In addition to the above five-cue model, we also included physical shaft length, Pressey's C measure (Pressey \& Murray, 1976), interaction terms 
from the experimentally defined stimuli, and dummy codes for the main effects (Pedhazur, 1982). These analyses were designed to see if nonlinearities in the relations between cues and either the environment or judgments attenuated the observed correlations and to explore the possible role of more complexly defined cues. These analyses failed to add to those to be discussed.

The main result of this analysis was that judgments of shaft length for Mueller-Lyer figures correlated more highly with judgments of shaft length for no-fin stimuli than they did with the physical shaft length of the MuellerLyer figures themselves $(r=.65$ vs. $r=.60$ for fins-in stimuli; $r=.59$ vs. $r=.47$ for fins-out stimuli, pooled over solid vs. dot stimuli). That is, subjective length judgments of the Mueller-Lyer stimuli were slightly more predictable from judgments of a finless shaft of the same length than they were from the actual length of the shaft corresponding to that figure.

Regardless of the model chosen, shaft length, either judged or physical, tended to have the highest zero-order correlation with judgments. This means that subjects were primarily responding to the veridical aspects of the stimuli. The next most strongly utilized cue, in terms of this definition, was ITD ( $r=.41$ for fins in and $r=.29$ for fins out). Fin angle and $C$ correlated to some extent with judgments of fins-in stimuli ( $\mathrm{r}=.25$ and .28 , respectively), but did not correlate with judgments of fins-out stimuli ( $r=$ -.03 and .06). Fin length and the $\mathrm{H}$ measure had minimal correlations with judgments in both conditions, the largest correlation being .17 .

Because the various predictors of judgments are not independent of one another, these zero-order correlations with the criterion are not the only measure of their importance as cues (cue utilization). We also determined the beta weights and uniquenesses (reduction in $\mathbf{R}^{2}$ following deletion of the variable), but the same general trends emerged. As Wilkinson (1975) noted, this need not always be the case.

The major goal of the study was to look for an invariant regression equation across subjects and conditions, which is evidence for a "critical stimulus." The equation need not be the same for fins-in and fins-out stimuli, since different critical stimuli could be the reason that $\mathrm{Co}$ ren et al. (1976) found them to be separable illusions, as we did.

We did not find an invariant equation of this type. There was no evidence for one "best" regression model which held even for a single subject in one condition, let alone across subjects.

One subject, chosen at random, illustrates the converse point that numerous regression models accounted for the structure of cue utilization equally well. We conducted an "all possible subsets" regression analysis plus Pressey's $\mathrm{C}$ measure), using fin-in responses. Four of the 15 possible two-variable models produced values of $R^{2}$ between .62 and $.64 ; 13$ of 20 three-variable models produced values of $R^{2}$ between .63 and $.65 ; 13$ of 15 four- variable models produced values of $R^{2}$ between .64 and .66 , and all 6 five-variable models produced values of $\mathrm{R}^{2}$ that ranged from .66 to .67 . This gradual increase in values of $R^{2}$ with the number of predictors simply reflects the mathematically defined increase in a multiple correlation as the number of predictors increases (Nunnally, 1978, pp. 179-180. Overall, the results indicate that no one set of cues is "best" in simulating the subject's behavior. As long as some measure of shaft length is included to reflect the veridical aspects of judging Mueller-Lyer stimuli, various subsets of cues that were equally effective in the simulation could be used.

This state of affairs is not surprising to someone familiar with the problem of obtaining a multiple regression equation. The point is that there are numerous highly correlated cues present in the Mueller-Lyer task, and this high degree of intercorrelation (multicollinearity) is what precludes the establishment of a unique equation. This is not, however, an artifact of the method, but a neglected truth about the stimulus environment to which the subject responds. Averaging over subjects to provide greater stability to the data did not alter this conclusion.

One further attempt was made to identify individual consistencies of judgment by examining whether the cue configurations people employed for fins-in stimuli were similar to the ones they used for fins-out stimuli. This involved a cluster analysis with Johnson's (1976) "maximum" method, as applied to the saturated (six-cue) structure of: (1) zero-order correlations, (2) beta weights, and (3) uniquenesses, in separate analyses. Regardless of the measure used for clustering, we failed to find a relationship between clusters obtained in the two conditions. In other words, subjects who had similar cue utilization structures in one condition did not necessarily have a similar cue utilization structure in the other.

\section{Field Independence Analysis}

The GEFT scores ranged from 5 to 18 (the maximum obtainable), with a mean of 12.3 and a standard deviation of 3.7. These figures are similar to those reported by Weitkin et al. (1971). The GEFT scores did relate to certain aspects of performance. Specifically, GEFT scores were positively related to the knowledge $(G)$ parameter of the lens analysis $(r=.48$ and $.41, \mathrm{p}<.01$ and .05 , for fins-in and fins-out conditions, respectively). This indicates that field-independent subjects were able to base their judgments more upon the cue structure than were field-dependent subjects. These GEFT scores did not correlate with the judgmental linearity (cognitive control, or $\mathbf{R}^{\mathrm{s}}$ ) measure.

\section{DISCUSSION}

Our main points of discussion are the similarities found (1) between the parametric results we obtained using magnitude estimation and the results previously obtained using more traditional psychophysical methods, and 
(2) between the correlational results we obtained with geometric illusions and those previously obtained in a variety of other contexts.

The method of magnitude estimation did produce the basic Mueller-Lyer effect. In addition, our finding that the expansion obtained with fins-out stimuli was greater than the contraction obtained with fins-in stimuli is consistent with findings of Adam and Bateman (1980), Christie (1975), and Heymans (1896). We found that increasing fin length increased the illusion with both finsin and fins-out stimuli. This is consistent with the findings of Lewis (1909) and Restle and Decker (1977). The latter investigators also reported that the fin-length effect was more pronounced and nonmonotonic with fins-out effect which is what we obtained.

The manipulation of fin angle produced linear changes in the amount of illusion for fins-in stimuli, and smaller, non-monotonic changes for fins-out stimuli, which is what Sekuler and Erlebacher (1971) noted. Those investigators also reported that their fins-in figure was more sensitive to ITD changes than their fins-out figure. We noted a difference in the fin length $\times$ fin angle interaction as a function of fin direction. Decreasing fin angle caused a greater increase in illusion magnitude with fins-in stimuli when the figures had long fins. This is an ITD effect, because changing angle causes a larger corresponding change in ITD at longer fin lengths.

The fin length $\times$ fin angle interaction was more complex for fins-out stimuli. Increasing fin angle produced a small, monotonic decrease in illusion magnitude for figures with short ( 3 or $8 \mathrm{~mm}$ ) fins, but had a larger, inverted U-shaped effect for figures with larger (13 or $8 \mathrm{~mm}$ ) fins. This is consistent with Pressey's concept of the "attentive field" in determining the effectiveness of context elements (Pressey \& Bross, 1973). The tips of the fins and, hence, the context created between the fins is increasingly likely to fall outside the attentive field at long fin lengths and acute angles.

The most unusual result was that changing fin angle had little effect when either the fin or shaft was short. This suggests that ITD plays a greater role with longer stimuli. When the shaft is short relative to the fins, ITD becomes so small that there is a qualitative change in the appearance of the figure. The shaft appears almost completely enclosed in a diamond-shaped surround so that ITD may be ignored or suppressed because of a tendency towards closure. That is, subjects tended to perceive stimuli with small physical ITDs as diamonds, making the apparent ITD zero.

Although there was no effect of stimulus type, there was an interaction with fin angle; the dot form was less sensitive to this manipulation than was the solid drawing. Erlebacher and Sekuler (1969) found a small, but significant, role of fin angle independent of its role in determining ITD. Consequently, our findings suggest that this additional effect may be structural, that is, due to image blur or retinal inhibition, since the effect is diminished when there are no intersecting lines.
We also found a fin length $\times$ fin angle interaction with solid forms but not with dot forms. This suggests a decreased influence of ITD on the dot forms, which we failed to find reported elsewhere. This effect is difficult to explain in terms of the decrese in intersecting line segments, since ITD effects are normally not interpreted in terms of retinal inhibition.

We did not replicate Griest-Bousquet and Schiffman's (1981) finding that fins-in dot forms had an opposite finangle effect and no fin-length effect. Their result may be an artifact of their use of a six-point dot form, which may have induced illusion-destroying grouping effects.

The main finding of our correlational analyses was that we failed to find evidence for a "critical stimulus" in that we could not derive a regression equation that was unique to an individual within or over conditions, let alone possessed generality across subjects and conditions. This is, of course, only one line of evidence, but it is consistent with Coren and Girgus's (1978) conclusion, which followed from their review of the literature. This conclusion does not rest upon the modification we made in the lens model, although it is based upon the more general assumptions made in regression analysis. The main reason that there was no "best" regression equation is that the stimulus environment is multicollinear, that is, it is extremely rich in highly correlated cues, regardless of the experimenter's attempts to derive a set of orthogonal independent variables.

Einhorn et al.'s (1979) comment on how the statistician's difficulty in determining a "best" equation under such circumstances mirrors the subject's difficulty in dealing with this rich stimulus environment was based upon data derived from clinical judgments and other nonpsychophysical data. Nonetheless, it seems extremely appropriate here. The problem of cue intercorrelation exists whether the experimenter chooses to recognize them explicitly, as we did, or not.

The regression analysis did provide information of a more positive nature. As one would reasonably expect, it did indicate that subjects were primarily attending to the veridical component of their stimulus environment, shaft length, and its subjective representation (judged nofin length) provided a slightly better definition than did its physical representation, a fairly common result (Monahan \& Lockhead, 1977). The analysis also confirmed the role of ITD for fins-in stimuli, which parallels Sekuler and Erlebacher's (1971) parametric results. Furthermore, fin angle also seems to play a more important role in determining responses to fins-in as opposed to fins-out stimuli, but the reverse is true for fin length. Conversely, the vertical distance from the tip of a fin to its shaft $(\mathrm{H})$ was not an important cue. This does contrast with Adam and Bateman's (1980) results. Their results may have been affected by illusion decrement since they presented each test form over 300 times per subject.

The fact that fins-in and fins-out stimuli were sensitive to different manipulations in both the parametric and correlational sense is consistent with the findings of several 
previous studies (Coren et al. 1976; Porac \& Coren, 1981; Sekuler \& Erlebacher, 1971). Indeed, it was the number of studies and diversity of methods by which this conclusion was reached that led us to avoid trying to fit the two sets of fin-direction data to the same equation. Differences between solid and dot forms were more subtle. These, of course, had to be fit to different equations, since the manipulation took place between subjects.

On the whole, our data do suggest that both fin-direction and stimulus-type effects are due to different mechanisms. One can, however, note that Pressey's assimilation theory may provide a single-process explanation. This theory attributes the illusion to assimilation of the shaft with the various contextual elements (Pressey, 1967). The "contextual-element-producing" portion of the assimilation process is probabilistic in nature, and the probability of the various elements' creating an illusion-inducing context depends upon various factors. This view is highly consistent with our multiple-cue approach. Pressey and Murray (1976) demonstrated the importance of the placement of elements in the attentive field. Our results further suggest the importance of "grouping" and "closure" as factors. The ITD became ineffective as a cue when the fin tips were close to one another, because closure overcame the normal tendency to assimilation; ITD was ignored as a consequence.

Our lens analysis provided a couple of additional points to consider. These points rest upon more specific assumptions than does the conclusion reached about the diffuseness of cue utilization. First, we found that the five specific cues incorporated into the analysis were generally sufficient predictors, even though not always necessary, since they were not utilized in a given analysis, for example, fin angle for fins-out stimuli. That is, most subjects were well represented by a linear model, as inferable from the fact that only five subjects failed to provide $\mathbf{R}_{\mathbf{s}}$ values that were at least .70. This figure is even higher than that reported by Rappoport (cited in Hammond et al., 1964) in a different type of three-cue task with $R_{e}$ of .99 . The essentially linear nature of his task makes it more comparable to our task than many other multiple-cue studies.

Despite the general linearity of the subject's judgments, 10 subjects had $\mathrm{C}$ values of .4 or greater. This indicates that these subjects were responding to cues that were different, but at least partially veridical, from those being used by the remaining subjects. Differences between these subjects and those described above, who did respond in terms of the postulated cues, are parallel to the individual differences found by Hake et al. (1967) in their task. In both tasks, subjects achieved similar performance levels using different strategies, a view quite at opposition to the critical-stimulus hypothesis.

Our results also tentatively suggest that the illusion is not so much due to such factors as retinal inhibition, which limit the subjects' knowledge. Rather, the illusion seems due to imperfect cognitive control (failure to rely upon one's knowledge). In other words, the subject has veridical information but fails to use it. Hammond and Sum- mers (1972) have provided parallel examples from clinical judgments. Indeed, this study suggests that the reasons that people misuse information about geometric figures are the same as the reasons that they misuse information about more cognitive and less perceptual events. Procedures designed to assess strategies used to achieve coherence thus seem applicable to both.

The distinction between performance deficits produced by lack of knowledge and by misapplication of that knowledge was given futher construct validation by the GEFT results. The measure of FI correlated with G (knowledge) but not with $R_{s}$ (application of knowledge). Since this measure has been shown to assess the ability to isolate a relevant figure embedded in a misleading context, the fact that it correlates with Mueller-Lyer judgments is not surprising by itself. The important point is the specific way it relates to knowledge and not its application (cognitive control).

In sum, our results suggest that the geometry of an illusion such as the Mueller-Lyer places a fundamental limit upon the degree to which its "critical" features may be specified. The present results, based upon lens model and related mulivariate analysis, in conjunction with more conventional parametric analyses, agree with Coren and Girgus's (1978) conclusions and are one line of evidence against the critical-stimulus hypothesis.

\section{REFERENCES}

ADAM, J., \& BAtEMAN, L. (1980). Control stimuli in investigations of the acute-angled and obtuse angled Mueller-Lyer illusions. Perception, 9, 476-474.

Bernstein, I. H., Fisicaro, S. A., Fox, J. A. (1976). Metacontrast suppression and criterion content: $A$ discriminant function analysis. Perception \& Psychophysics, 20, 198-204.

Bernstein, I. H., SMITH, D. B., \& ADEY, M. (1977). Perceptual and response interdependencies in visual masking. In S. Dornic \& A. F. Sanders (Eds.), Attention and performance, VI (pp. 177-194). Hillsdale, N. J.: Erlbaum.

BREITMEYER, B. , \& GANZ, L. (1976). Implications of sustained and transient channels for theories of visual pattern masking, saccadic suppression, and information processing. Psychological Review, 83, 1-36.

Brunswick, E. (1952). Conceptual framework of psychology. Chicago: University of Chicago Press.

BRUnswick, E. (1956). Perception and the representative design of experiments (2nd. ed.). Berkeley: University of California Press.

Christie, P. S. (1975). Assymetry in the Mueller-Lyer illusion: Artifact of genuine effect? Perception, 4, 453-457.

Coren, S., \& Girgus, J. S. (1978). Seeing is decieving: The psychology of visual illusions. Hillsdale, N. J.: Erlbaum.

Coren, S., Girgus, J. S., Ehruchman, H., \& Hakstian, A. R. (1976). An empirical taxonomy of visual illusions. Perception \& Psychophysics, 20, 129-137.

Einhorn, H. J., Kleinmuntz, D. N., \& Kleinmuntz, B. (1979). Linear regression and process-training models of judgment. Psychological Review, 86, 465-485.

Erlebacher, A., \& SeKuler, R. (1969). Explanation of the MuellerLyer illusion: Confusion theory examined. Journal of Experimental Psychology, 80, 462-467.

Erlebacher, A., \& Sekuler, R. (1974). Perceived length depends on exposure duration: Straight lines and Mueller-Lyer stimuli. Journal of Experimental Psychology, 103, 724-728.

Eysenck, H. J. \& Slater, P. (1958). Effects of practice and rest on fluctuations in the Mueller-Lyer illusion. British Journal of Psychology, 49, 246-256. 
GolDBERG, L. R. (1970). Man versus model of man: A rationale, plus some evidence for a method of improving on clinical inferences. Psychological Bulletin, 73, 422-432.

Griest-Bousquet, S. \& Schiffman, H. R. (1981). The manyu illusions of the Mueller-Lyer. Perception, 10, 147-154.

Hake, H. W., Faust, G. W., McIntyre, J. S., \& Murray, H. G. (1967). Relational perception and models of perceiver operations. Perception \& Psychophysics 2, 489-498.

Hake, H. W., Rodwan, A., \& Weintraub, D. (1966). Noise reduction in perception. In K. R. Hammond (Ed.), The psychology of Egon Brunswik. New York: Holt, Rinehart \& Winston.

Hammond, K. R., Hursch, C. J., \& Todd, F. J. (1964). Analyzing the components of clinical inference. Psychological Review, 71, $438-456$.

Hammond, K. R., \& Summers, D. A. (1972). Cognitive control. Psychological Review, 79, 438-456.

HeYMANS, G. (1896). Quantitative Untersuchungen über das optiche Paradoxen. Zeitschrift für Psychologie, 9, 221-225.

JoHnson, S. C. (1967). Hierarchical clustering schemes. Psychometrika, 32, 241-252.

Kaufman, L. R., \& Richards, W. (1969). Spontaneous fixation tendencies for visual forms. Perception \& Psychophysics, 5, 85-88.

LEWIS, E. O. (1908). The effect of practice on the perception of the Mueller-Lyer illusion. British Journal of Psychology, 2, 294-306.

LEwIs, E. O. (1909). Confluxion and contrast effects in the MuellerLyer illusion, British Journal of Psychology, 3, 21-41,

MonahaN, J. S., \& LockHEAD, G. R. (1977). Identification of integral stimuli. Journal of Experimental Psychology: General, 106, 94-110.

Nunnally, J. D. (1978). Psychometric theory. New York: McGraw-Hill.

Pedhazur, E. J. (1982). Multiple regression in behavioral research (2nd. ed.). New York: Holt, Rinehart and Winston.

PorAC, C., \& CoREN, S. (1981). Life-span age trends in the perception of the Mueller-Lyer: Additional evidence for the existence of two illusions. Canadian Journal of Psychology, 35, 58-62.
Pressey, A. W. (1967). A theory of the Mueller-Lyer illusion. Perceptual and Motor Skills, 25, 641-644.

Pressey, A. W., Bross, M. (1973). Assimilation theory and the reversed Mueller-Lyer illusion. Perception, 2, 211-217.

Pressey, A. W., \& MurRay, R. (1976). Further developments in the assimilation theory of geometric illusions: The adjacency priciple. Perception \& Psychophysics, 19, 536-544.

Restle, F., DeCKer, J. (1977). Size of the Mueller-Lyer illusion as a function of its dimensions: Theory and data. Perception \& Psychophysics, 21, 489-503.

RichaRDS, W., \& KaUFMAN, L. R. (1969). "Center-of-gravity" tendencies for fixation and flow patterns. Perception \& Psychophysics, $5,81-84$.

SEKUler, R., ERlebacher, A. (1971). The two illusion sof MuellerLyer: Confusion theory re-examined. American Journal of Psychology, 84, 477-481.

STEVENS, S. S. (1962). The surprising simplicity of sensory metrics. American Psychologist, 17, 29-39.

Szucko, J. J., \& KLEINMUNTZ, G. (1981). Statistical versus clinical lie detection. American Psychologist, 56, 488-496.

TUCKER, L. (1964). A suggested alternative formulation in the developments by Hursch, Hammond and Hursch, and by Hammond, Hursch and Todd. Psychological Review, 71, 528-530.

VIRSU, V. (1971). Tendencies to eye movement and misperception of curvature, direction, and length. Perception \& Psychophysics, 9, 65-72.

WILKINSON, L. (1975). Response variable hypotheses in the multivariate analysis of variance. Psychological Bulletin, 82, 408-412.

Witkin, H. A., Oltman, P., RAskin, E., KARP, S. A. (1971). Manual for the Group Embedded Figures Test. Palo Alto, CA: Consulting Psychologists Press.

(Manuscript received November 14, 1983; revision accepted for publication July 23, 1984.) 